\title{
Discriminative Analysis of Brain Function at Resting-State for Attention-Deficit/Hyperactivity Disorder
}

\author{
C.Z. Zhu' ${ }^{1}$, Y.F. Zang ${ }^{1}$, M. Liang ${ }^{1}$, L.X. Tian ${ }^{1}$, Y. He ${ }^{1}$, X.B. $\mathrm{Li}^{1}$, M.Q. Sui ${ }^{2}$, \\ Y.F. Wang ${ }^{2}$, and T. Z. Jiang ${ }^{1}$ \\ ${ }^{1}$ National Laboratory of Pattern Recognition, Institute of Automation, \\ Chinese Academy of Sciences, P.R. China \\ czzhu@nlpr.ia.ac.cn \\ ${ }^{2}$ Institute of Mental Health, Peking University, P.R. China
}

\begin{abstract}
In this work, a discriminative model of attention deficit hyperactivity disorder (ADHD) is presented on the basis of multivariate pattern classification and functional magnetic resonance imaging (fMRI). This model consists of two parts, a classifier and an intuitive representation of discriminative pattern of brain function between patients and normal controls. Regional homogeneity (ReHo), a measure of brain function at resting-state, is used here as a feature of classification. Fisher discriminative analysis (FDA) is performed on the features of training samples and a linear classifier is generated. Our initial experimental results show a successful classification rate of $85 \%$, using leave-one-out cross validation. The classifier is also compared with linear support vector machine (SVM) and Batch Perceptron. Our classifier outperforms the alternatives significantly. Fisher brain, the optimal projective-direction vector in FDA, is used to represent the discriminative pattern. Some abnormal brain regions identified by Fisher brain, like prefrontal cortex and anterior cingulate cortex, are well consistent with that reported in neuroimaging studies on ADHD. Moreover, some less reported but highly discriminative regions are also identified. We conclude that the discriminative model has potential ability to improve current diagnosis and treatment evaluation of ADHD.
\end{abstract}

\section{Introduction}

Attention-deficit/hyperactivity disorder (ADHD) is one of the most commonly diagnosed childhood behavioral disorders. According to related reports, 3 6\% American and 5\% Chinese school-age children are affected by ADHD. Developmentally inappropriate inattention, impulsivity, and hyperactivity are three core symptoms of ADHD. Children with ADHD have difficulty on controlling their behaviors or focusing their attentions which result in an adverse effect on academic performance and social function. Current available diagnosis and treatment evaluation of ADHD are mainly made from the levels of the core symptoms. Ranking of the symptoms is usually made by the parents or teachers of the children, which is unfortunately subjective. Therefore more objective approaches are highly desired. 
With the high spatial and temporal resolution as well as the non-invasive advantage, structural and functional magnetic resonance imaging (MRI) have been playing an increasingly important role in brain studies. Volumetric [1], morphological [2, 3] and functional [4, 5] brain properties have been studied on ADHD with MRI [6, 7]. Voxel-based structural and functional MRI studies on ADHD have suggested various brain abnormalities [7]. However such a group-level statistical difference is less helpful to diagnosis. Currently some promising studies on mental diseases $[8,9,10,11$, 12] with multivariate statistical classifiers using neuroimaging information were reported. Unfortunately few were concerned on ADHD. Moreover, explanation of classification result in these studies is still unsatisfactory. In this work, an ADHD discriminative model which includes an ADHD classifier and an intuitive representation of discriminative pattern is proposed on the basis of Fisher discriminative analysis (FDA) of brain function obtained from resting-state fMRI.

The classification feature and classification algorithm will be detailed in Section 2 and 3 respectively. Materials are presented in Section 4. Experimental results and discussion are provided in Section 5. Section 6 is devoted to conclusion and some further directions.

\section{Mapping of Brain Function at Resting-State}

Low-frequency $(0.01-0.08 \mathrm{~Hz})$ fluctuation (LLF) synchrony among motor cortices was studied by Biswal at 1995, which indicated that LFF was physiologically meaningful [13]. Later some studies about diseases had been conducted using LFF synchrony $[14,15,16]$. As a mapping of brain function, regional homogeneity (ReHo) was first proposed to measure the regional synchrony of LFF voxel by voxel, and then employed to verify the default mode network (DMN) [17] successfully in [18]. ReHo has also been used to locate the ROIs automatically without a priori in a study of brain function connectivity [19]. Here, we used ReHo derived from fMRI series scanned at resting-state as a feature of classification. ReHo was defined at a given voxel as the temporal similarity of the LFF between the voxel and its neighbors, which was calculated with Kendall's coefficient as (1).

$$
W=\frac{\sum\left(R_{i}\right)^{2}-n(\bar{R})^{2}}{\frac{1}{12} K^{2}\left(n^{3}-n\right)}
$$

where $\mathrm{W}$ is the Kendall's coefficient among given voxels, $\mathrm{R}_{\mathrm{i}}$ the sum rank of the ith time point, $\bar{R}=((n+1) K) / 2$ the mean of the $\mathrm{R}_{\mathrm{i}}{ }^{\prime} \mathrm{s}, \mathrm{K}$ the number of time series within a measured cluster and we used $\mathrm{K}=27$ as in [18].

\section{Pseudo-Fisher Discriminative Analysis}

Fisher discriminative analysis (FDA) is a widely used technique in the domain of pattern recognition $[20,22]$. Suppose there are two classes of samples with features of dimension D. Here, D is defined as the number of voxels in consideration. FDA is used to find a projective direction, $\omega^{*} \in \mathfrak{R}^{\mathrm{D}}$, along which the two classes of projected 
samples are separated with maximal ratio of between-class distance and within-class variability. Mathematically, objective function (2) is to be maximized

$$
J(\omega)=\frac{\omega^{T} S_{b} \omega}{\omega^{T} S_{w} \omega}
$$

where

$$
S_{w}=\sum_{i=1}^{N_{1}}\left(x_{i}^{1}-m_{1}\right)\left(x_{i}^{1}-m_{1}\right)^{T}+\sum_{i=1}^{N_{2}}\left(x_{i}^{2}-m_{2}\right)\left(x_{i}^{2}-m_{2}\right)^{T} \text {, }
$$

and

$$
S_{b}=\left(m_{1}-m_{2}\right)\left(m_{1}-m_{2}\right)^{T}
$$

are between-class scatter matrix and within-class scatter matrix respectively; $\left.x_{i}^{1}\right|_{i=1} ^{N_{1}}$ and $\left.x_{i}^{2}\right|_{i=1} ^{N_{2}}$ are feature vectors, $m_{1}$ and $m_{2}$ mean feature vectors, $\mathrm{N}_{1}$ and $\mathrm{N}_{2}$ sample sizes. Theoretically, the optimal $\omega^{*}$ can be determined by:

$$
\omega^{*}=S_{w}^{-1}\left(m_{1}-m_{2}\right)
$$

However, in case of small-sample $\left(\mathrm{N}_{1}+\mathrm{N}_{2}<<\mathrm{D}\right)$, which is common in brain image analysis, computing of inverse matrix of $\mathrm{S}_{\mathrm{w}}$ is an ill-posed problem and therefore FDA would yield an unreliable result. While pseudo-Fisher discriminative analysis (pFDA), which is a variation of classical FDA, can solve the problem by using the pseudo-inverse of $S_{w}$ to substitute the inverse of $S_{w}[23,24]$. Briefly, principal component analysis (PCA) was firstly applied on sample features, $\mathrm{x}_{\mathrm{i}} \in \mathfrak{R}^{\mathrm{D}}$, to find a linear subspace, $\mathfrak{R}^{\mathrm{d}}$, spanned by all the eigenvectors, $\left\{\alpha_{1}, \alpha_{2}, \ldots, \alpha_{\mathrm{d}}\right\}$, with non-zero eigenvalue. Representing an original sample feature, $x \in \mathfrak{R}^{\mathrm{D}}$, with $\left\{\alpha_{1}, \alpha_{2}, \ldots, \alpha_{d}\right\}$ would result in a low-dimension feature, $y \in \mathfrak{R}^{\mathrm{d}}\left(\mathrm{d}=\mathrm{N}_{1}+\mathrm{N}_{2}-1\right)$, so that classical FDA and (3) can be directly used in the subspace to find $\omega^{*} \in \Re^{\mathrm{d}}$. Projecting each sample, $\mathrm{y} \in \mathfrak{R}^{\mathrm{d}}$, onto $\omega^{*} \in \mathfrak{R}^{\mathrm{d}}$ can result in a one-dimensional score of $z \in \mathfrak{R}^{1}$ by inner product operation, $z=\left\langle y, \omega^{*}\right\rangle$. Finally, the classification threshold, $\mathrm{z}_{0} \in \mathfrak{R}^{1}$, was determined by:

$$
z_{0}=\left(N_{1} m_{1}^{z}-N_{2} m_{2}^{z}\right) /\left(N_{1}+N_{2}\right)
$$

where $m_{1}^{z}$ and $m_{2}^{z}$ are centers of projective scores of the two classes.

\section{Materials}

To eliminate the impact of head motion on the calculation of ReHo maps, the subjects with head motion greater than $1.2 \mathrm{~mm}$ or rotation greater than $1.2^{\circ}$ were excluded. The remained 9 ADHD and 11 controls (age range of 11-15 years and IQ > 80) were used for further analysis.

The imaging processes were undertaken on the SIEMENS TRIO 3-Tesla scanner in Institute of Biophysics, Chinese Academy of Sciences. For each subject we concerned the following two sets of imaging data: resting-state fMRI time cou rses and 3D structural MRI. Echo Planer Imaging (EPI) Blood Oxygenation Level Dependent (BOLD) images were acquired axially with the following parameters: $2000 / 30 \mathrm{~ms}$ (TR/TE), 30 slices, $4.5 / 0 \mathrm{~mm}$ (thickness/gap), $220 \times 220 \mathrm{~mm}$ (FOV), $64 \times 64$ (resolution), $90^{\circ}$ (flip angle), the whole session lasted for 480 seconds. 3D spoiled gradient- 
recalled whole-brain volume was acquired sagittally with the following parameters: 1700/3.92 ms (TR/TE), 192 slices, 1.0/0 mm (thickness/gap), $256 \times 256 \mathrm{~mm}$ (FOV), $256 \times 256$ (resolution), $12^{\circ}$ (flip angle).

Preprocessing procedures for fMRI signals included motion correction, withinsubject registration, time aligning across slices, time series linear detrending, voxels resampling to $3 \times 3 \times 3 \mathrm{~mm}^{3}$, spatially smoothing $(\mathrm{FWHM}=4 \mathrm{~mm})$ and spatial normalization. All these processes were undertaken using SPM2 [25].

\section{Experiments Results and Discussion}

pFDA was performed on ReHo maps of the 9 ADHD and 11 controls, and an ADHD classifier and a representation of discriminative pattern were generated. First, the classifier was tested with the training samples to indicate the separability of the classifier on training set. Then leave-one-out (LOO) cross validation approach was employed to estimate the prediction ability of the model. Classification results are listed in the top row of Table 1, form which zero training error is achieved, and correct predictions performed on ADHD and controls are $78 \%$ and $91 \%$ respectively. The total correct prediction rate reaches $85 \%$.

Table 1. Classification results

\begin{tabular}{|l|l|c|c|c|c|}
\hline \multicolumn{2}{|c|}{ Discriminative model } & Training set & \multicolumn{3}{c|}{ LOO test correct rate } \\
\cline { 4 - 6 } & & correct rate & Controls & ADHD & Total \\
\hline $\begin{array}{l}\text { Functional } \\
\text { information }\end{array}$ & ReHo Map & $100 \%$ & $91 \%$ & $78 \%$ & $85 \%$ \\
\hline $\begin{array}{l}\text { Structural } \\
\text { information }\end{array}$ & Intensity & $100 \%$ & $38 \%$ & $67 \%$ & $53 \%$ \\
\cline { 3 - 6 } & Morphology & $100 \%$ & $50 \%$ & $56 \%$ & $53 \%$ \\
\hline
\end{tabular}

The distribution of projective scores of both the training and predicting samples in a 20-round LOO test are shown in Fig. 1, where white circles and squares represent normal controls and children with ADHD in the training set of LOO test respectively, and black circles and squares represent the control and patient for predicting respectively. The crosses indicate the corresponding classification thresholds determined by (4). As in Fig. 1, there are only one testing control and two testing patients located on the wrong sides of the classification boundary by the classifier. Moreover, withinclass variations of projective scores of training samples are all close to zero, and between-class distances are quite large. This result convinces the objective of FDA.

With $\left\{\alpha_{1}, \alpha_{2}, \ldots, \alpha_{\mathrm{d}}\right\}$, the optimal projective direction in subspace $\Re^{\mathrm{d}}$ can be easily inversely mapped to the original space $\mathfrak{R}^{\mathrm{D}}$. The projective direction in $\mathfrak{R}^{\mathrm{D}}$ or Fisher brain, as a part of the discriminative model, was used to visualize the discriminative pattern of ReHo between the children with ADHD and normal controls. As illustrated in Fig.2, the larger the amplitude (positive or negative) of a voxel in the Fisher brain, the more the voxel contributes to the final discrimination. As shown in Fig.3, some highly discriminative regions are identified by the Fisher brain. Among these regions, prefrontal cortex and anterior cingulate cortex have been reported to be involved in 


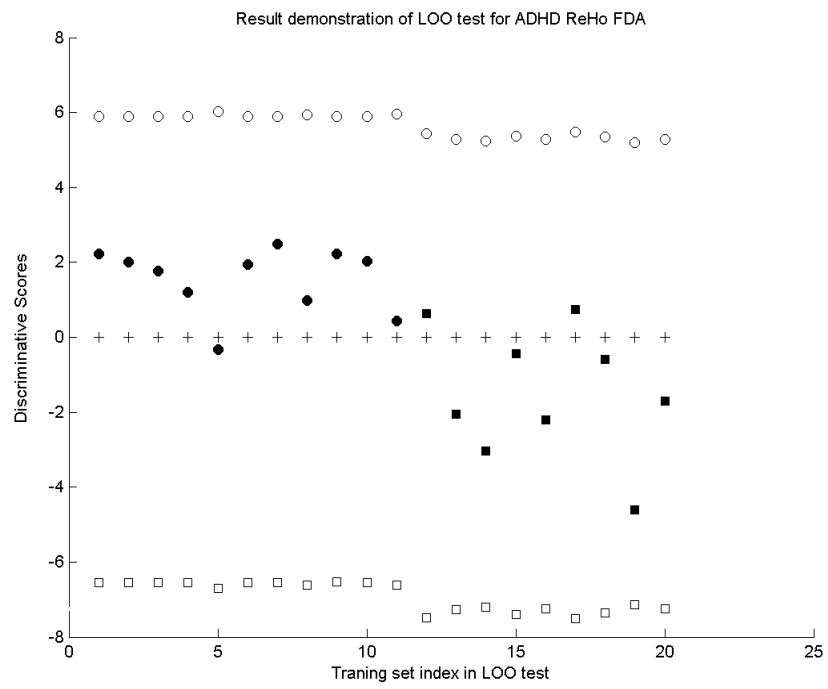

Fig. 1. Distribution of discriminative scores in LOO test

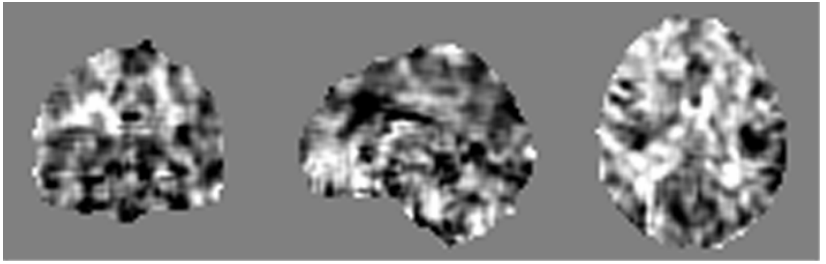

Fig. 2. Fisher brain to visualize the discriminative pattern

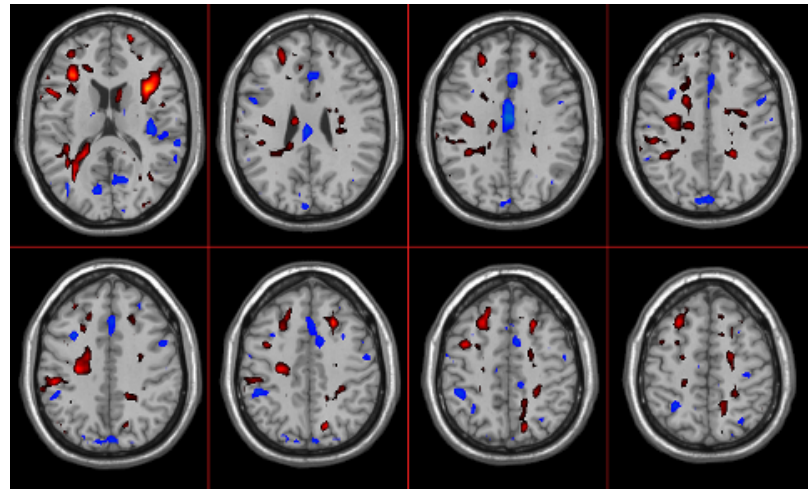

Fig. 3. Highly discriminative regions identified by Fisher brain. Red: ADHD>control; Blue: ADHD<control. 
higher brain functions like attention and inhibition and abnormal for ADHD subjects $[1,2,4,7]$. Moreover, some less reported but highly discriminative regions are also identified.

Our classifier was compared with other two typical linear classifiers, Batch Perceptron [20] and linear support vector machine (SVM) [21]. Table 2 lists the classification results of the three methods. Considering the stochastic property of Batch Perceptron algorithm, we repeated the 20-round LOO test for 10 times, each with a random initialization. Then the correct rates of 10 times of LOO tests were averaged as the final result of Batch Perceptron. From Table 2 we see that Batch Perceptron (54\%) hardly yields a meaningful result. Though linear SVM has much better performance than Batch Perceptron, its classification rate $(80 \%)$ is obviously lower than that of the proposed classifier $(85 \%)$.

Table 2. Comparison of different linear classifer

\begin{tabular}{|l|c|c|c|}
\hline \multirow{2}{*}{ Methods } & \multicolumn{3}{|c|}{ LOO test correct rate } \\
\cline { 2 - 4 } & Controls & ADHD & Total \\
\hline Batch Perceptron & $62 \%$ & $46 \%$ & $54 \%$ \\
\hline Linear SVM & $100 \%$ & $56 \%$ & $80 \%$ \\
\hline Proposed & $91 \%$ & $78 \%$ & $85 \%$ \\
\hline
\end{tabular}

To compare the discriminative ability of functional brain information with that of structural brain information, FDA was also applied to 3D structural MR images. All 3D structural MR images were spatially normalized using SPM2 [25]. To comprehensively investigate the discriminative ability of structural brain information, both original 3D MR images and their tissue segmentation results were used as the classification features. Multi-context fuzzy clustering (MCFC) algorithm was used for tissue segmentation since it is insensitive to the intensity inhomogeneities [26]. The classification results of FDA using original MR images and segmented images are depicted in the second and third row of Table 1 respectively. Generally speaking, no significant difference in classification performance appears between intensity based approach and morphology based one, and none of them yield a meaningful classification result (53\%). The results clearly demonstrate that ReHo map, as a mapping of brain function of resting state, is more effective for discrimination of ADHD than structural information of either the original MR intensity or the tissue segmentation result. More importantly, this implies that brain function may be more susceptible than brain structures for ADHD.

\section{Conclusion}

In this paper, a discriminative model of ADHD was proposed on the basis of Fisher discriminative analysis of ReHo map derived from fMRI scanned at resting state. The validation of the method was verified by experimental results. Compared with various classifiers and classification features, our method achieved much better classification performance. Furthermore, it yielded a significant representation for the discriminative pattern of brain function between the children with ADHD and the normal con- 
trols. Potential improvement of the diagnosis and treatment evaluation of ADHD can be realized based on the evidence given by the results of the discriminative model.

Evaluation of the proposed method with larger sample size and multi-center imaging data of children with ADHD is considered in future work. The statistic property of the Fisher brain is another issue to be addressed. Moreover, those less reported but highly discriminative regions found in this study will be further examined.

Acknowledgement. This work was partially supported by the Natural Science Foundation of China, Grant No. 30425004, the National Key Basic Research and Development Program (973) Grant No. 2003CB716103, and Natural Science Foundation of China, Grant No. 30470575.

\section{References}

1. S.H. Mostofsky, K.L. Cooper, and W.R. Kates, et al. Smaller prefrontal and premotor volumes in boys with attention-deficit/hyperactivity disorder. Biol Psychiatry 52(8):785-794, 2002.

2. E.R. Sowell and P.M. Thompson et al. Cortical abnormalities in children and adolescents with attention deficit hyperactivity disorder. THE LANCET, 362: 1699-1707, 2003.

3. P.C. Berquin, J.N. Giedd, L.K. Jacobsen, S.D. Hamburger, and A.L. Krain, et al. Cerebellum in attention-deficit hyperactivity disorder: a morphometric MRI study. Neurology 50:1087-1093, 1998.

4. G. Bush, J. A. Frazier, S. L. Rauch, and L. J. Seidman, et al, Anterior Cingulate Cortex Dysfunction in Attention Deficit/Hyperactivity Disorder Revealed by fMRI and the Counting Stroop. BIOL PSYCHIATRY 45:1542-1552, 1999.

5. C.J. Vaidya, G. Austin, G. Kirkorian, H.W. Ridlehuber, J.E. Desmond, G.H. Glover, and J.D. Gabrieli. Selective effects of methylphenidate in attention deficit hyperactivity disorder: A functional magnetic resonance study. Proc Natl Acad Sci U S A, 95:14494-14499, 1998.

6. M. Semrud-Clikeman, R.J. Steingard, P. Filipek, J. Biederman, K. Bekken, and P.F. Renshaw. Using MRI to examine brain-behavior relationships in males with attention deficit disorder with hyperactivity. J Am Acad Child Adolesc Psychiatry, 39:477-484, 2000 .

7. L.J. Seidman, E.M. Valera, and G. Bush. Brain function and structure in adults with attention-deficit/hyperactivity disorder. Psychiatr Clin N Am, 27: 323-347, 2004.

8. P. Golland, W. E. L. Grimson, M. E. Shenton, and R. Kikinis, Deformation Analysis for Shape Based Classification.,In Proc. IPMI'2001, LNCS 2082, pp. 517-530

9. Z.Q. Lao, D.G. Shen, Z. Xue, B. Karacali S. M. Resnick, and C. Davatzikosa, Morphological classification of brains via high-dimensional shape transformations and machine learning methods, NeuroImage, 21 (2004) 46-57.

10. Y.X Liu. L. Teverovskiy, and O. Carmichael et al., Discriminative MR Image Feature Analysis for Automatic Schizophrenia and Alzheimer's Disease Classification, MICCAI 2004, LNCS 3216, pp. 393-401,2004.

11. J. Stoeckel, N. Ayache, and G. Malandain et al., Automatic Classification of SPECT Images of Alzheimer's Disease Patients and Control Subjects. MICCAI 2004, LNCS 3217, pp. 654-662, 2004. 
12. D. Kontos, V. Megalooikonomou, and D. Pokrajac, et al., Extraction of Discriminative Functional MRI Activation Patterns and an Application to Alzheimer's Disease, MICCAI 2004, LNCS 3217, pp. 727-735, 2004.

13. B.B. Biswal, F.Z. Yetkin, V.M. Haungton, J.S. Hyde. Functional connectivity in the motor cortex of resting human brain using echo-planar MRI, Magn Reson Med., 34:537-541, 1995.

14. M.J. Lowe, B.J. Mock and J.A. Sorenson. Functional connectivity in single and multi,slice echoplanar imaging suing resting-state fluctuation, NeuroImage, 7:119-132. 1998.

15. D. Cordes, V.M. Haughton and K. Arfanakis et al. Mapping functionally related regions of brain with functional connectivity MR imaging. AJNR Am J Neuroradiol, 21:1636-1644. 2000.

16. S.J. Li, Z. Li, G.H. Wu, M.J. Zhang, M. Franczak and P.G. Antuono. Alzheimer Disease: Evaluation of a Functional MR Imaging Index as a Marker. Radiology, 225:253-259, 2002.

17. M. E. Raichle, A. M. MacLeod, A. Z. Snyder, W. J. Powers, D. A. Gusnard, and G. L.Shulman. A default mode of brain function. Proc. Natl. Acad. Sci. USA 98,676-682, 2001

18. Y.F. Zang, T.Z. Jiang et al,. Regional homogeneity approach to fMRI data analysis. NeuroImage, 22: 394-400 2004.

19. Y. He, Y.F. Zang, T.Z. Jiang and M. Liang, Detecting Functional Connectivity of the Cerebellum Using Low Frequency Fluctuations (LFFs). MICCAI 2004, LNCS 3217, pp. 907-915, 2004.

20. R. Duda, P. Hart, and D. Stork. Pattern Classification. John Wiley \& Sons, New York, 2001.

21. V.N. Vapnik. Statistical Learning Theory. J. Wiley, New York. 1998.

22. P. N. Belhumeur, J. P. Hespanha and D. J. Kriegman. Eigenfaces vs. Fisherfaces: Recognition Using Class Specific Linear Projection. IEEE Trans. PAMI 19: 711-720, 1997.

23. S. Raudys and R.P.W. Duin. Expected classification error of the Fisher linear classifier with pseudo-inverse covariance matrix.Pattern Recognition Letters, 19:385-392, 1998.

24. J. Yang and J.Y. Yang. Why can LDA be performed in PCA transformed space? Pattern Recognition 36,563 - 566, 2003.

25. K. J. Friston, A. P. Holmes and K. J. Worsley et al., Statistical parametric maps infunctional imaging: A general approach. Hum. Brain Mapp., 2:189-210, 1995.

26. C.Z. Zhu and T. Z. Jiang. Multicontext fuzzy clustering for separation of brain tissues in magnetic resonance images. NeuroImage, 18(3): 685-696, 2003. 